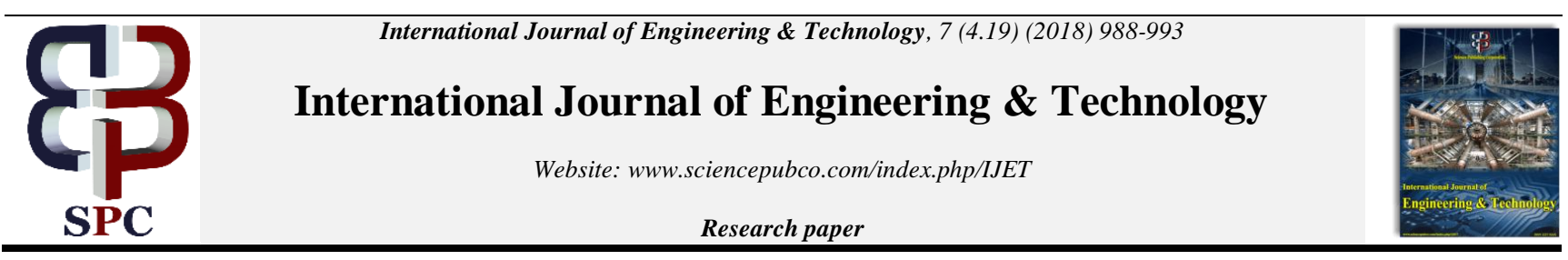

\title{
A Heuristic Local Search Algorithm for Improving Cluster and Routing Stability in Sensor Networks
}

\author{
L. Manoharan', A.Ezil Sam Leni ${ }^{2}$ \\ ${ }^{1}$ Research Scholar, Sathyabama Institute of Science and Technology, Chennai \\ ${ }^{2}$ Professor \& Head, Department of CSE Jeppiaar SRR Engineering College,

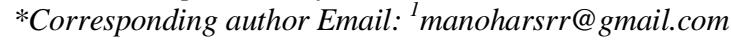

\begin{abstract}
Route selection using optimal nodes in Wireless Sensor Network (WSN) is a prominent task to improve network performance without deterioration. Intrinsic characteristics of the node, network traffic are some of the reason where routing algorithms fail to prove their consistency. In such cases, the conventional routing schemes must be equipped with additional network management features to retain network performance. This manuscript proposes a Heuristic Local Search (HLS) algorithm for improving routing efficiency of WSN. This search algorithm is backboned by clustering process; the cluster head is elected by satisfying balanced network metrics: energy and distance. Congestion preventive routing considers the same objective function in selecting an outside intermediate node that mitigates packet loss due to congestion. Implication of similar objective function under different constraints streamlines routing and prolongs the operation period of the cluster head. The process of the proposed HLS is evaluated using extensive simulation for the metrics: throughput, network lifetime, cluster head changes, energy utilization and packet loss ratio.
\end{abstract}

Keywords: Cluster Head Selection, Congestion Control, Local Search, Objective Function, Route Optimization.

\section{Introduction}

Wireless Sensor Network (WSN) integrates a wide-range of sensors that gathers information from the environment and conveys to a sink node [1]. The sensor devices/ nodes are electrical hardware components multi-functional ability. Sensor operations like sensing and communication; etc is powered by built-in limited capacity batteries [3]. Due to ease of deployment, sensor nodes are directed to sense and actuate milieu changes like temperature, object locations, pressure, etc [2]. A conventional technique to conserve energy and to improve routing is data gathering that is renowned in WSN [4]. With the growing communication demand, sensor node support is extended to both cloud and non-cloud applications. The applications of sensor are distributed over commercial and non-commercial automations, defense, habitat monitoring, health-care industry, etc [5].

The intrinsic resource constraint nature of the sensor nodes confines the operations of the nodes to a certain limit. This hinders both routing and sensing ability of the node besides computational and storage operations. An external factor that affects the operations of the sensor node is the region of placement. Alongside the different design issues the nodes rely on their neighbors for information sensing and processing. Due to dependency, power expenditure grows high, leading to earlier energy drain of the nodes. In this scenario, the node needs to be replaced or its battery has to be recharged at the earliest to pursue on-going communication [6]. Meanwhile, prolonging the lifetime of the nodes needs to be focused to sustain communication despite of energy failures.
In the recent years, energy optimization and design of energy efficient routing protocols have emerged for addressing the forementioned issue.

Routing protocols are designed to achieve energy conservation, data aggregation and network lifetime prolonging besides routing [7].

Another conventional technique for achieving energy efficiency in WSN is clustering. Clustering is the process of grouping like attributed nodes together for a harmonized communication. Cluster Head $(\mathrm{CH})$ serves as the control authority for integrating other nodes communication. The network is segregated into clusters, each of the cluster are intended to perform different operations. Clustering encompasses the entire network through integrated data gathering model, facilitating data collection from different network locations. The $\mathrm{CH}$ identifies the optimal path based on distance to relay the aggregated data to the sink node [9, 11]. Cluster head accepts multiple communications at a same time and to prevent congestion, it allocates time-slots for communication for its member nodes. In a clustering process, communicating nodes alone remain in active state, the rest of the nodes are directed to move to sleep state. This process of state change-over is aided through duty-cycle algorithms designed for sensor nodes. This is more predominant in improving energy efficiency by curtailing unnecessary energy expenses $[8,10]$.

\section{Related Work}

Devi et al., [12] proposed protocol named energy efficient selective opportunistic routing (EESOR) an extended version of energy efficient opportunistic routing (EEOR). 
Simulation results represent that proposed protocol is reliable in terms of packet delivery ration, improves network lifetime and reduces delay.

Leu et al., [13] proposed a new clustering protocol namely regional energy aware clustering with isolated nodes (REAC-IN), an extension of LEACH protocol which considers the residual energy and regional energy of the nodes in selecting the cluster head. The distance between isolated node and sink is calculated and that determines whether the isolated communicate data with sink or cluster head in previous round. On simulation proposed protocol outperforms network lifetime and provides stability to the network.

Jesus et al., [14] studied various distributed data aggregation methods which includes a) structured approach is a dependent based approach which follows certain network topologies and routing protocols. The structured approach is further classified as hierarchy based and ring based b) unstructured approach are independent which is applicable to any network topology and routing protocols. This approach is classified as flooding, random walk and gossip based approaches and c) hybrid based approach is a combination of both structured and unstructured approach which overcomes the drawbacks of both the approaches. The hybrid based approach is further classified as hierarchy and gossip based approaches.

Wang and Liu [15] analyzed the issues by comparing wired and wireless sensor networks data collections and studied wireless sensor network data collections which have been classified into three phases namely a) Deployment stage is considered to achieve coverage with minimal number of nodes and to improve network connectivity based on (i) deployment for area coverage and (ii) deployment for location coverage. b) Data delivery approaches are considered to provide efficient communication between source and sink and to optimize QoS parameters such as (i) reliability (ii) latency (iii) throughput and (iv) energy consumption. c) Control message dissemination stage denotes propagation of control messages with low time and communication cost to enhance network on the basis of residual energy with parameters such as (i) flooding and gossiping (ii) different enhancements and (iii) Integrated with duty cycle.

Xie and Jia [16] proposed a clustering method using hybrid compressive sensing an analytical method which optimizes network lifetime. On simulation results show that our proposed method can reduce transmission when compared with previous methodologies and can even equally perform in homogeneous network with irregular sensor field.

Petrioli et al., [17] proposed ALBA-R (Adaptive Load Balancing Algorithm - Rainbow) a cross layer mechanism which selects relay nodes for load balancing and solves routing issues around a dead end. On simulation proposed algorithm shows tremendous results on comparison with previous available techniques with lower energy and overhead.

Ren et al., [18] proposed an Energy Balanced Routing Protocol (EBRP), which considers the depth, energy density and residual energy of the node to make packets flow towards the sink with minimal amount of energy. On simulation, EBRP performances better in energy balancing and in prolonging network lifetime.

The authors of [19] proposed an ant colony variant EAACA that considers shortest distance neighbor and energy consumption in a balanced manner. Nodes with average lesser energy consumption are selected for routing in the shortest path.

Camilo et. al [20] projected an Ant Colony Optimization based EEABR algorithm to improve the networks' energy efficiency. EEABR routes packets to the sink node through the updates of backward ants. The backward ants upda nodes' energy level and distance pheromone update and the source selects an optimal path among the set of ant updates. The authors in [11] propose LEACH for minimizing energy dissipation and to improve network lifetime. In LEACH, a cluster head is responsible for data aggregation inside the cluster and relaying outside the cluster. The cluster head is selected randomly in a stochastic manner, to minimize unnecessary energy dissipation caused due to independent node communication. [21] and [22] defaces in managing heterogeneous energy constraints and to overcome the drawback, the authors of [23] proposed an Energy Aware Routing Protocol (EAP) for energy efficient cluster head selection. In EAP, the cluster head is selected based on the ratio of higher residual energy compared to the mean available energy of the nodes. EAP forms a spanning tree using all the cluster heads the root node communicates to the sink in one-hop, energy consumed in free space is less.

\section{Contributions}

The contributions of the manuscript are as follows:

i. Design of an objective based clustering process for improving cluster stability and prolonging the operation time of the cluster head. The factors distance and energy are considered for satisfying the defined objective function.

ii. Congestion Preventive routing process wherein the traffic concentrated routes are identified and failures are repaired by redirecting the transmission. The same objective for identifying cluster head is used to identify intermediate node to minimize computational complexity.

iii. Performance assessment of the proposed algorithm through appropriate simulations for verifying the consistency of the proposed algorithm. The simulation results and their description demonstrate the likelihood of the proposed algorithm.

\section{Heuristic Local Search (HLS) Algorithm}

\subsection{Network Model}

We consider a WSN in a $X \times Y$ region with $\left\{\mathrm{n}_{1}, \mathrm{n}_{2}, \ldots \mathrm{n}_{\mathrm{n}}\right\} \in \mathrm{N}$ sensor nodes. The $\mathrm{N}$ nodes are dispersed in a random manner. The nodes are grouped to form clusters where a Cluster Head $(\mathrm{CH})$ server as the control authority. The responsibilities of a $\mathrm{CH}$ are: data gathering, neighbor selection and transmission. In order to support routing, Cluster Member (CM) is deliberated to facilitate outside node communication. The information transmitted from the $\mathrm{CH}$ is received at the sink node (SN). Figure 1 illustrates the network model of the proposed system.

\section{Assumptions}

i. The initial $\mathrm{CH}$ will be the communication initiating sensor node.

ii. Not all the nodes are grouped into clusters. This shows the presence of Intermediate Nodes (IN) in the network.

iii. The mobility of the nodes has a lesser impact on the network performance

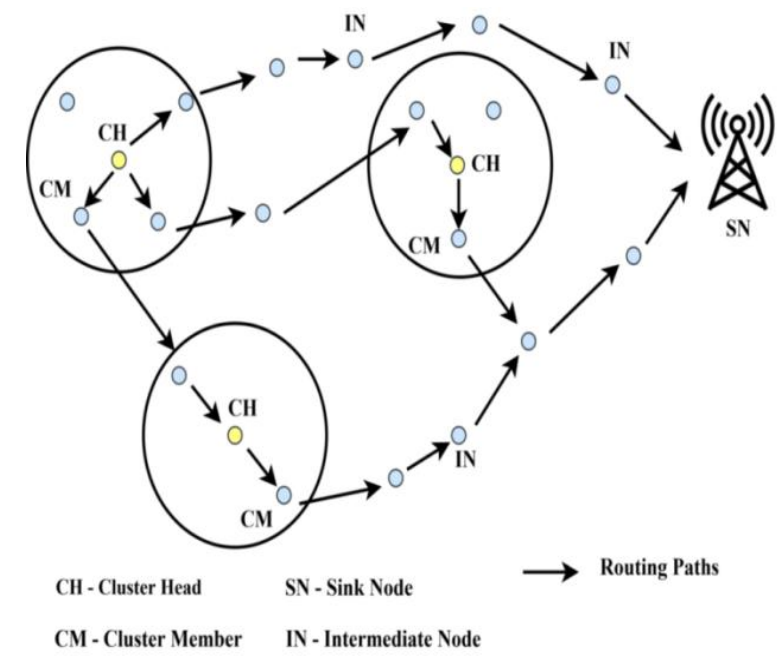

Fig. 1: Network Model 


\subsection{Energy Model}

Let $E_{\text {init }}, E_{T}$ and $E_{R}$ denote the initial, transmitting and receiving energy of a sensor node. Then the energy utilization $\left(E_{U}\right)$ is estimated using equation (1)

$\mathrm{E}_{\mathrm{U}}=\mathrm{E}_{\mathrm{T}}+\mathrm{E}_{\mathrm{R}}$

Similarly, $E_{T}$ and $E_{R}$ are estimated using equations (2) and (3)

$E_{T}=d r_{t} * e_{t} * t_{t}$

And

$\mathrm{E}_{\mathrm{R}}=\mathrm{dr}_{\mathrm{r}} * \mathrm{e}_{\mathrm{r}} * \mathrm{t}_{\mathrm{r}}$

Where,

$d r_{t}$ and $d r_{r}$ are the transmitting and receiving rates of the data, $e_{t}$ and $e_{r}$ are the transmitting and receiving energies and $t_{t}$ and $t_{r}$ are the transmitting and receiving time, correspondingly.

Let $E_{r e s}$ represent the residual energy of the node that is given by

$\mathrm{E}_{\mathrm{res}}=\mathrm{E}_{\text {init }}-\mathrm{E}_{\mathrm{U}}$

\subsection{Methodology}

The concept of the proposed local search is aided by clustering process. Therefore, the proposed method is modeled to operate in both clustering and routing process. Figure 2 illustrates the overall process of the proposed HLS.

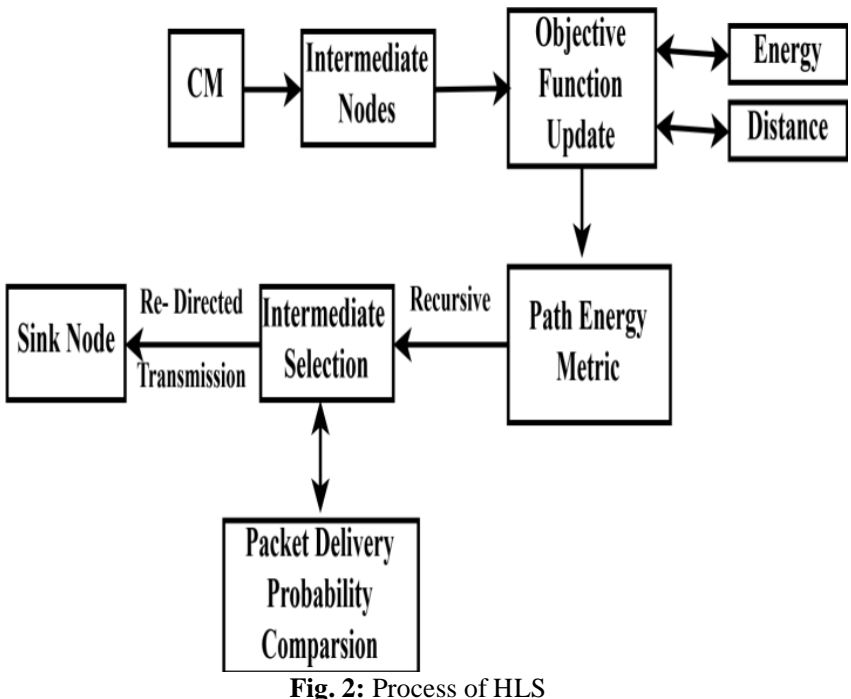

The proposed method is of two planes: Stabilized Cluster Head Selection and Congestion preventive routing. The first plane is responsible for electing a $\mathrm{CH}$ that sustains for a prolonged time. This minimizes the $\mathrm{CH}$ selection complexity. Congestion preventive routing is designed to confine traffic over the available paths.

\subsubsection{Stabilized CH Selection}

This plane is designed to minimize cluster head changes period over time. Frequent change in $\mathrm{CH}$ determinates network performance by increasing delay and communication hold we consider the energy of the $\mathrm{CH}$ and the distance to the sink node for internal and external stability process. Let $\mathrm{f}(\mathrm{n})$ be the objective function that is to be achieved. The object function is defined using remaining energy and distance to the sink. Equation (5) represents the object function as $\mathrm{f}(\mathrm{n})=\mathrm{w}_{1} * \mathrm{E}_{\mathrm{res}}+\mathrm{w}_{2} * d(\mathrm{n}, \mathrm{SN})$
Where $w_{1}$ and $w_{2}$ are the prioritized weight factors, $d(n, S N)$ is the distance from node $n$ to sink node. Here $n \in C M$ if the $\mathrm{CH}$ relies on its members for routing. The objective function must satisfy the maximum packet delivery by evaluating energy of the path. For a path $\mathrm{p}$, the energy parameters $\left(\Delta \mathrm{E}_{\mathrm{p}}\right)$ is estimated as

$\Delta \mathrm{E}_{\mathrm{p}}=\left(\frac{\mathrm{E}_{\mathrm{res}}(\mathrm{n})-\mathrm{l}_{\mathrm{p}}}{\mathrm{d}(\mathrm{n}, \mathrm{SN})}\right)$

Where $l_{p}$ is the load observed over the path $p$. equation (5) needs to satisfy $\Delta \mathrm{E}_{\mathrm{p}}$ for all transmission from a node $\mathrm{n}$. A node that is retains (5) controlled under (6) is selected as the $\mathrm{CH}$.

Let $t_{\text {inif }}$ and $t_{\max }$ denote the initial and maximum time of a node to satisfy (5). If $t_{a}$ is the active time of a CH then, $t_{\text {inif }}<t_{a} \leq$ $t_{\max }$. The second $\mathrm{CH}$ is selected by observing the second maximum $t_{a}$ of a sensor node $n$. From the equation (6), the weights are adjustable; this will select different nodes as $\mathrm{CH}$, in the different time intervals. The above discussion from equation (5) is analyzed under three cases for node selection. The node selection can be either inside or outside the cluster i.e. $\mathrm{CH}$ or an IN selection.

Case 1: The priority for energy is greater than the distance i.e. $\mathrm{w}_{1}>\mathrm{w}_{2}$

Analysis 1: If the above case is true, then the node with higher residual energy is opted for transmission. The distance factor here is given lesser priority and therefore, this is suitable in selecting a $\mathrm{CH}$. The $\mathrm{CH}$ will be additional one-hop away from the sink as the process takes place within a cluster. If the above condition is exploited for an intermediate node, then the time of packet delivery would increase. This is because the distance from the redirecting node to the sink is unknown.

Case 2: Distance priority is higher than energy $\mathrm{w}_{2}>\mathrm{w}_{1}$

Analysis 2: If the distance priority is higher than the energy, then this objective is utilized for intermediate selection. Despite energy, distance factor is prioritized for the nodes that conjoin transmissions in a united manner. To prevent transmission delay and to improve packet delivery probability, the weight factor of the intermediate node for distance can be higher than energy. The routing will be a conventional process provided, the node must ensure $\max \left(\frac{\mathrm{E}_{\mathrm{res}}(\mathrm{n})-\mathrm{l}_{\mathrm{p}}}{\mathrm{d}(\mathrm{n}, \mathrm{SN})}\right)$.

Case 3: The priority for both factors is alike i.e. $\mathrm{w}_{1}=\mathrm{w}_{2}$

Analysis 3: This balances the constraints of intermediate and $\mathrm{CH}$ selection with equal consideration of the considered metrics. The node selection (as an intermediate or an $\mathrm{CH}$ ) will be optimal in determining both distance factor and energy metric so as to improve stability in cluster formation as well as routing. This case is suitable for a few initial rounds of the network as the energy ceases considerably after a few transmissions over time. The constraint that needs to be attained is either the $\mathrm{CH}$ or the $\mathrm{IN}$, needs to satisfy $\max \left(\frac{E_{\text {res }}(n)-l_{p}}{d(n, S N)}\right)$ for achieving $f(n)$.

\subsubsection{Congestion Preventive Routing}

Congestion preventive Routing comes into existence when a specific routing path is congested due to route error or link failure at the time of transmission. In such cases, the neighbor transmits a Route Error message to the $\mathrm{CH}$ and $\mathrm{CH}$ initiates re-routing from the very first node. This increases the rate of congested transmission retarding of the $\mathrm{CH}$ node. The traffic in the error route is redirected to the non-familiar route where the flow rate is increased. To avoid this, HLS seeks the aid of objective function. The objective function designed in selecting a $\mathrm{CH}$ is utilized for the intermediate nodes to select the proper routing paths. It initiates local congestion preventive routing through the node that satisfies equation (5) confined to equation (6). Through congestion preventive routing, the predecessor node discovers less flow routes to the sink node. The distance factor is also considered to ensure longest path discoveries. 
Objective function satisfying equation (6) is achieved by the CMs; the energy alone is not sufficient to ensure successful packet rerouting. The re-routing procedure requires attuning factor for ensuring successful packet delivery at the sink node. The attuning factor is constructed using the probability of packet delivery $\left(\rho\left(d_{r}\right)\right)$ at the sink node. If the sink is located ' $h$ ' hops away from the aggregator then, packet delivered at the sink is given by [24]

$\rho\left(d r_{r}\right)=\prod_{i=S N}^{S N+h-1}\left(1-\frac{d r_{t}-d r_{r}}{d r_{t}}\right)$

Where, $\frac{d r_{t}-d r_{r}}{d r_{t}}$ is the packet loss ratio

In path redirecting process, the $\mathrm{CH}$ adjoins the current transmission along with the intermediate retransmission. Here, the sequential placement of packets requires additional time for transmission. This time is less than the rerouting and retransmission process. Therefore, $t_{\text {inif }}<t_{a} \leq\left(t_{\max }+t_{r d}\right)$, where $t_{r d}$ is the redirecting time.

The congestion preventive path selection technique is used when a nodes' link fail due to maximum congestion. To prevent redirecting of route error and to perform congestion less transmissions, the autonomous routing feature of the nodes is relied. Therefore, prime function of a sensor node is to monitor forwarder energy and the path distance along with $\Delta \mathrm{E}_{\mathrm{p}}$. If the sensor node experiences congestion, it searches for new routing paths in a conventional manner. If link stability $\rho_{\mathrm{s}}$ decreases over the forward time interval $\{(\mathrm{t}+1),(\mathrm{t}+2), \ldots,(\mathrm{t}+\mathrm{q})\} \in \mathrm{t}$, the predecessor node discovers all possible routes to the sink in a conventional manner.

Let $\left\{\mathrm{n}_{1}, \mathrm{n}_{2}, \ldots, \mathrm{n}_{\mathrm{j}}\right\} \in \mathrm{N}$ be the one-hop direct neighbors for the congested node that requires redirection to the sink node. Let $f(\alpha)$ be the next neighbor selection function satisfying equation (6), then the selection function is given by equation (8)

$\mathrm{f}(\alpha)=\min \left\{\Delta \mathrm{E}_{\mathrm{p}_{1}}, \Delta \mathrm{E}_{\mathrm{p}_{2}}, \ldots, \Delta \mathrm{E}_{\mathrm{p}_{\mathrm{j}}}\right\}$

\subsection{Process of Congestion preventive Routing}

Figure 3 illustrates the process of Congestion Preventive Routing.

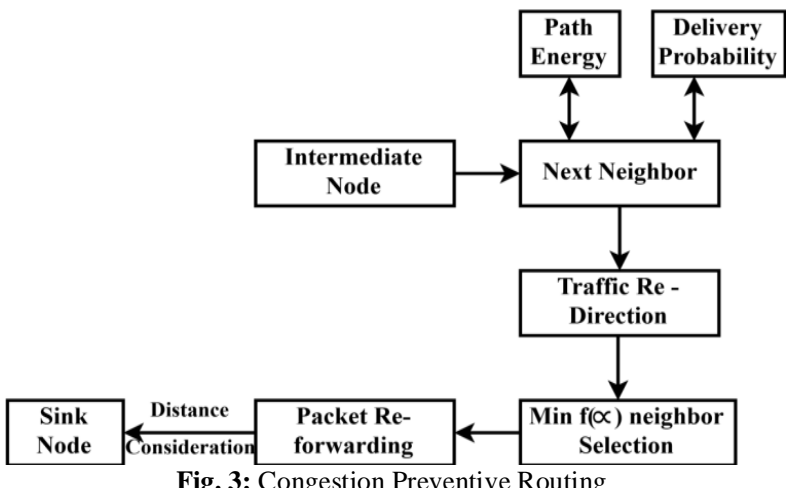

Fig. 3: Congestion Preventive Routing

The path node monitors the neighbor's $\Delta \mathrm{E}_{\mathrm{p}}$ and $\mathrm{d}(\mathrm{n}, \mathrm{SN})$. When a link failure occurs, path node initiates conventional routing broadcast to discover all available neighbors that redirects the current transmission to sink node from the failure point. This discovery phase is similar to $\mathrm{CH}$ selection process with to prevent additional computational complexities. For a congestion preventive route discovery, the path node generates a Route Request (RREQ) packet with its ID as the forwarding neighbor and Sink node ID. The direct neighbor replies with a Route Reply (RREP) confirming the path for transmission.
At the time of selecting a local neighbor for redirection, the path node considers the $\left(t_{\max }+t_{r d}\right)$ and $\rho\left(d_{r}\right)$ of the newly discovered nodes. The constraint that is to be satisfied is the any neighbor selected must be confined to equation (6). The process of congestion preventive routing is described in Algorithm1 and iteration process (begins from L1) will keep on executing until end of transmission.

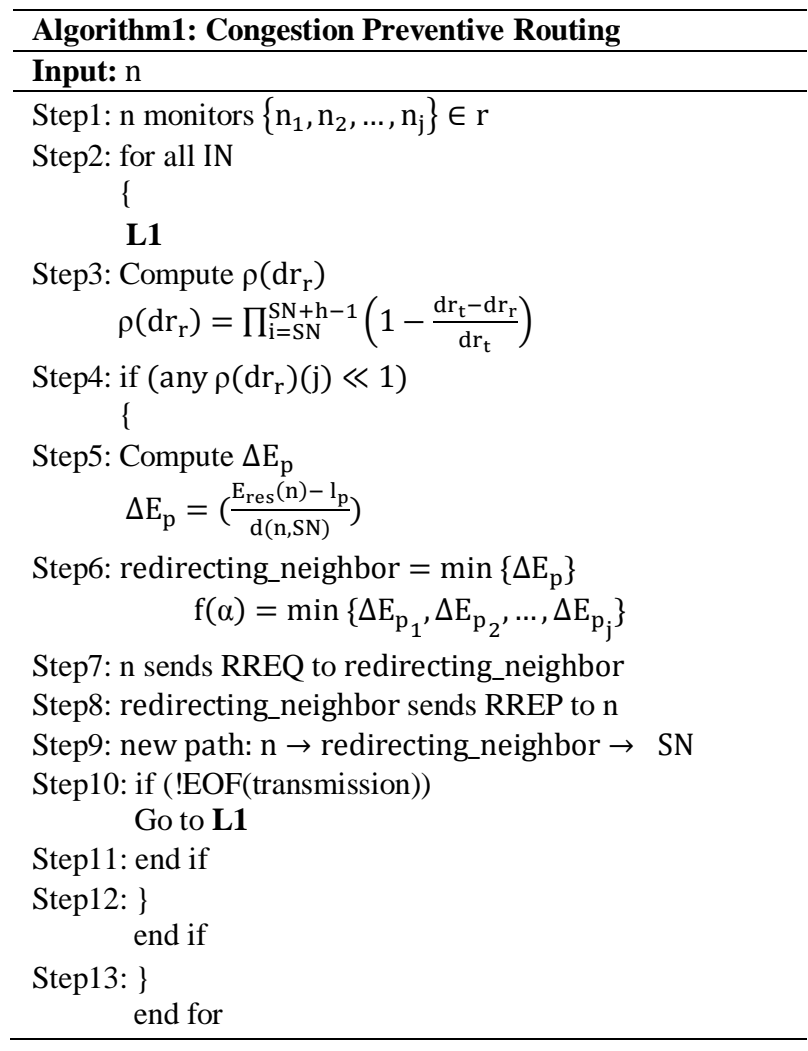

\section{Performance Evaluation}

The proposed Heuristic Local Search for routing enhancement in WSN is assessed using the following metrics: Throughput, network lifetime, Cluster Head Changes, Energy Utilization and Packet loss. The fairness of the proposed algorithm is compared with the existing EBRP [18] and Transmission Efficient Clustering (TEC) [16] for the above performance metrics. Table 1 shows the simulation parameters and its corresponding values.

Table 1: Simulation Parameters

\begin{tabular}{|l|l|}
\hline \multicolumn{1}{|c|}{ Parameter } & \multicolumn{1}{c|}{ Value } \\
\hline Network Region & $1000 \mathrm{~m} * 1000 \mathrm{~m}$ \\
\hline Sensor Nodes & 100 \\
\hline Communication Range & $250 \mathrm{~m}$ \\
\hline Protocol & AODV \\
\hline Number of Packets & 1500 \\
\hline Application Category & Constant Bit Rate \\
\hline Initial Energy & $20 \mathrm{~J}$ \\
\hline
\end{tabular}

\section{Analysis}

\section{Throughput}

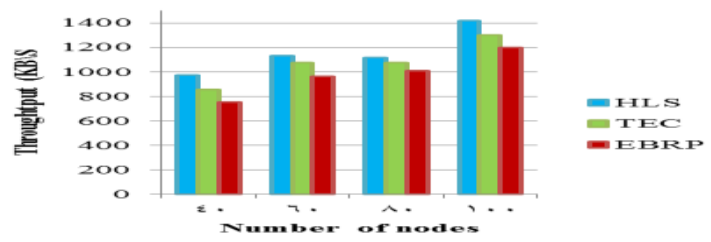

Fig. 4: Throughput Comparison 
Figure 4 compares the throughput achieved in the proposed HLS with the existing TEC and EBRP respectively. In the proposed HLS, both $\mathrm{CH}$ and IN are selected by evaluating the energy and distance metric individually. The nodes that are opted as $\mathrm{CH}$ and IN for routing and transmission needs to satisfy equation (6) proving their stability. The alternate intermediate nodes selected for traffic redirection ensures packet delivery if routed through the new node. Both energy and delivery factors are estimated over each node to endure successful packet delivery. This improves the network throughput of the proposed HLS.

\section{Network Lifetime}

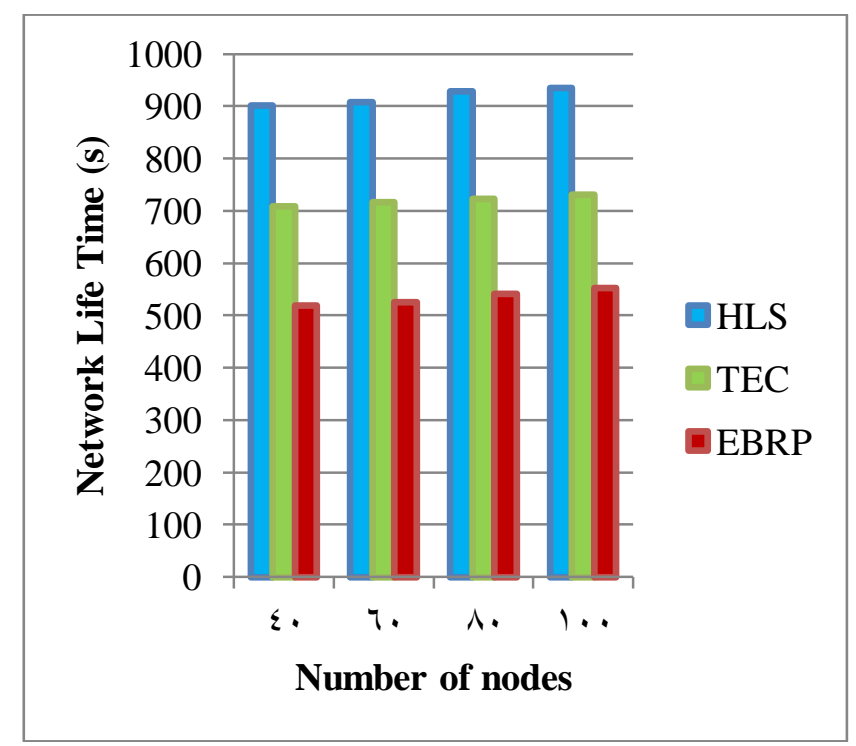

Fig. 5: Network Lifetime Comparison

Energy conservation improves network lifetime despite node density. In the proposed HLS, the number of transmissions and network operations are limited to certain sensor nodes. The sensor node achieving the objective function and satisfying equation (6) will remain in transmitting state and the rest will remain idle. Whenever congestion occurs, further transmissions are redirected to specific nodes other than multipath transmissions. This reduces unnecessary energy expenses of the nodes, retaining higher network lifetime (Figure 5).

\section{Cluster Head Changes}

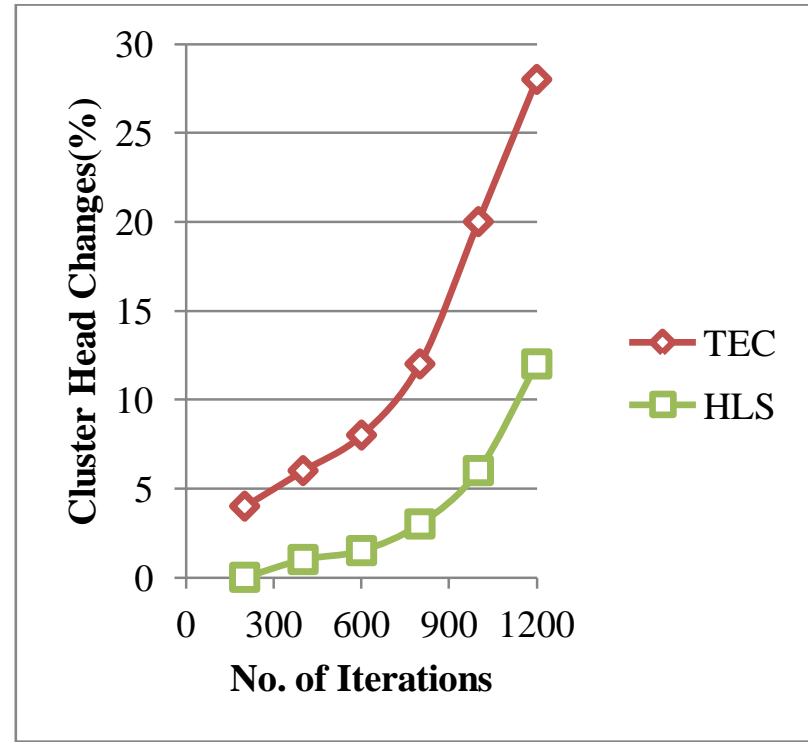

Fig. 6: Cluster Head Changes
Figure 6 illustrates the average cluster head changes compared between the existing TEC and the proposed HLS algorithm. In the proposed HLS, the cluster head is determined using balanced energy and distance metrics. The $\mathrm{CH}$ remains in its role until the $\mathrm{E}_{\mathrm{res}}<\frac{\Delta \mathrm{E}_{\mathrm{p}}}{\mathrm{n}}$, this condition remains until there is path availability from the inner CM to the sink node. It is not necessary that the $\mathrm{CH}$ needs to be replaced if its energy is less than any other $\mathrm{CM}$ as in TEC. The more the path availability, the higher is the $\mathrm{CH}$ stability. Moreover, the $\mathrm{CH}$ is also replaced if it does not satisfy equation (6), and this minimizes periodic header replacement.

\section{Energy Utilization}

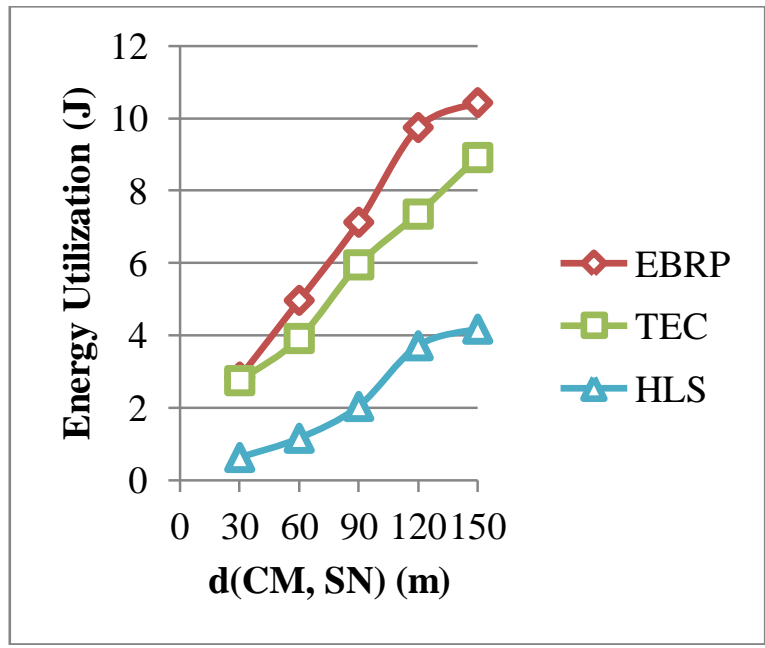

Fig. 7: Energy Utilization

Energy requirement boosts up in discovering new routes and $\mathrm{CH}$ changes other than transmission and reception. In the proposed $\mathrm{HLS}$, number of re-routing procedures and $\mathrm{CH}$ changes are less, minimizing periodic energy expenses. The overall energy expense of the network is shared between minimum number of nodes required for transmitting and receiving packets. Moreover, not all the available IN are induced for transmitting packets at the same time during congestion. A node more close to the $\mathrm{SN}$ is selected by evaluating its distance and energy. An IN with lesser path energy metric within permitted distance factor is alone selected for packet redirection. Therefore, the energy requirement of the network is less compared to the exiting methods (Figure 7).

\section{Packet Loss}

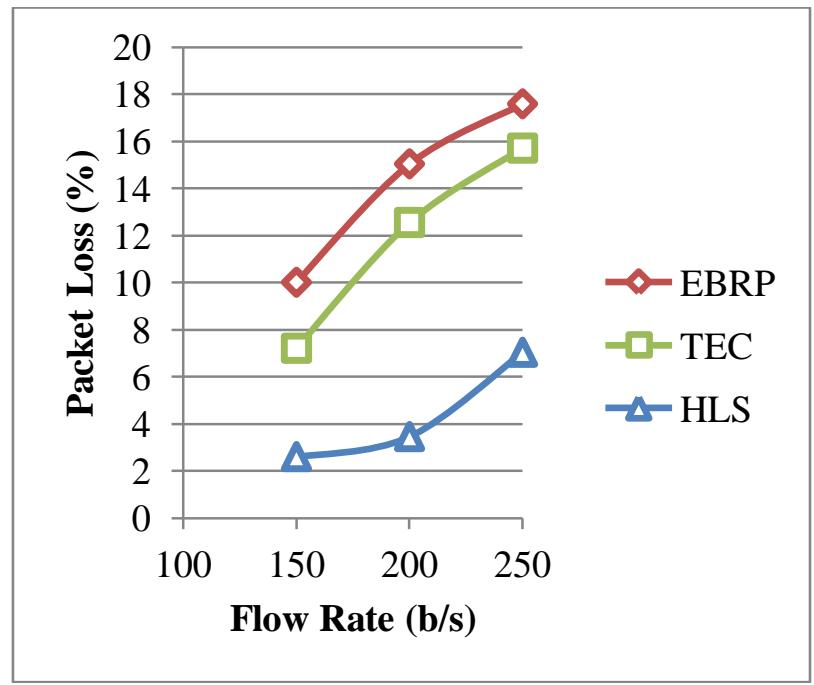

Fig. 8: Packet Loss Comparison 
Through a dedicated congestion preventive routing process aided by local search process, congestion in the network is evaded. The congestion preventive routing conjoins redirecting packets with the previously available links to the SN. Additional flow burden over a link with lesser $\Delta \mathrm{E}_{\mathrm{p}}$ is halted to control additional packet loss. Similarly, as the level of admit able traffic to the existing link is unknown, the probability of packet delivered at the sink is estimated for the available nodes. Higher is the $\rho\left(d_{r} r_{r}\right)$ value lesser is the packet loss factor. This feat is achieved in the proposed HLS (Refer Figure 8).

\section{Conclusion}

In order to improve the routing and to diminish congestion in WSN, this manuscript introduces a Heuristic Local Search Algorithm with a unified objective. The local search algorithm is aided by clustering process; the $\mathrm{CH}$ and intermediate node selection are facilitated by a common objective function. Traffic concentrated routes are identified and new routes are identified through nodes that achieve the objective. To strengthen redirected transmission, verification of end-to-end delivery probability at the sink ensures a streamlined transmission process. The performance of the proposed algorithm is verified using Throughput, network lifetime, cluster head changes, energy utilization and packet loss ratio.

\section{References}

[1] X. Liu, "Atypical hierarchical routing protocols for wireless sensor networks: A review," IEEE Sensors Journal, vol. 15, no. 10, pp. 5372-5383, October 2015.

[2] S. Ehsan and B. Hamdaoui, "A survey on energy-efficient routing techniques with QoS assurances for wireless multimedia sensor networks," IEEE Communications Surveys \& Tutorials, vol. 14 no. 2, pp. 265-278, Second Quarter 2012.

[3] N. A. Pantazis, S. A. Nikolidakis, and D. D. Vergados, "Energyefficient routing protocols in wireless sensor networks: A survey," IEEE Commun. Surveys Tuts., vol. 15, no. 2, pp. 551-591, 2nd Quart., 2013.

[4] Y. Mo, B. Wang, W. Liu, and L. T. Yang, "A sink-oriented layered clustering protocol for wireless sensor networks," Mobile Networks and Applications, vol. 18, no. 5, pp. 639-650, Oct 2013.

[5] Y. Zhang, M. Qiu, C.-W. Tsai, M. M. Hassan, and A. Alamri, "HealthCPS: Healthcare cyber physical system assisted by cloud and big data," IEEE System Journal, to appear 2015

[6] D. Zhang, G. Li, K. Zheng, X. Ming, and Z.-H. Pan, “An energy balanced routing method based on forward-aware factor for wireless sensor networks," IEEE Transactions on Industrial Informatics, vol. 10, no. 1, pp. 766-773, February 2014.

[7] J. Aweya, "Technique for differential timing transfer over packet networks," IEEE Transactions on Industrial Informatics, vol. 9, no. 1, pp. 325-336, February 2013.

[8] K. Kredo, II, and P. Mohapatra, "Medium access control in wireless sensor networks," Comput. Netw., vol. 51, no. 4, pp. 961-994, 2007.

[9] X. Gu, J. Yu, D. Yu, G. Wang, and Y. Lv, "Ecdc: An energy and coverage-aware distributed clustering protocol for wireless sensor networks," Computers \& Electrical Engineering, vol. 40, no. 2, pp. 384-398, February 2014.

[10] O. Younis and S. Fahmy, "HEED: a hybrid, energy-efficient, distributed clustering approach for ad hoc sensor networks," IEEE Transactions on Mobile Computing, vol. 3, no. 4, pp. 366-379, December 2004.

[11] M. M. Afsar, and M.-H. Tayarani-N, "Clustering in sensor networks: A literature survey,” J. Netw. Comput. Appl., vol. 46, pp. 198-226, Nov. 2014.

[12] C. R. Y. Devi, B. Shivaraj, S. H. Manjula, K. R. Venugopal, and L. M. Patnaik, "EESOR: Energy Efficient Selective Opportunistic Routing in Wireless Sensor Networks," Communications in Computer and Information Science Recent Trends in Computer Networks and Distributed Systems Security, pp. 16-31, 2014.

[13] J.-S. Leu, T.-H. Chiang, M.-C. Yu, and K.-W. Su, "Energy Efficient Clustering Scheme for Prolonging the Lifetime of
Wireless Sensor Network With Isolated Nodes," IEEE Communications Letters, vol. 19, no. 2, pp. 259-262, 2015.

[14] P. Jesus, C. Baquero, and P. S. Almeida, "A Survey of Distributed Data Aggregation Algorithms," IEEE Communications Surveys \& Tutorials, vol. 17, no. 1, pp. 381-404, 2015.

[15] F. Wang and J. Liu, "Networked Wireless Sensor Data Collection: Issues, Challenges, and Approaches," IEEE Communications Surveys \& Tutorials, vol. 13, no. 4, pp. 673-687, 2011.

[16] R. Xie and X. Jia, "Transmission-Efficient Clustering Method for Wireless Sensor Networks Using Compressive Sensing," IEEE Transactions on Parallel and Distributed Systems, vol. 25, no. 3, pp. 806-815, 2014.

[17] C. Petrioli, M. Nati, P. Casari, M. Zorzi, and S. Basagni, “ALBAR: Load-Balancing Geographic Routing Around Connectivity Holes in Wireless Sensor Networks," IEEE Transactions on Parallel and Distributed Systems, vol. 25, no. 3, pp. 529-539, 2014.

[18] F. Ren, J. Zhang, T. He, C. Lin, and S. K. D. Ren, "EBRP: Energy-Balanced Routing Protocol for Data Gathering in Wireless Sensor Networks," IEEE Transactions on Parallel and Distributed Systems, vol. 22, no. 12, pp. 2108-2125, 2011.

[19] D. Cheng, Y. Xun, T. Zhou, W. Li, “An energy aware ant colony algorithm for the routing of wireless sensor networks," Intelligent Computing and Information Science, Springer Berlin Heidelberg, 2011.

[20] T. Camilo, C. Carreto, J.S. Silva1, F. Boavida, "An EnergyEfficient Ant-Based Routing Algorithm for Wireless Sensor Networks," M. Dorigo, et al. (Eds.): ANTS 2006, LNCS 4150, pp. 49-59, 2006.

[21] Hu Gang, Xie Dongmei, Wu Yuanzhong. "Research and Improvement of LEACH for Wireless Sensor Networks", Chinese Journal of Sensors and Actuators, Vol. 20 No. 6, pp. 1 391-1 396, 2007.

[22] O. Younis and S. Fahmy, "HEED: a hybrid, energy-efficient, distributed clustering approach for ad hoc sensor networks," IEEE Transactions on Mobile Computing, vol. 3, no. 4, pp. 366-379, 2004.

[23] M. Liu, J. Cao, G. Chen, and X. Wang, “An Energy-Aware Routing Protocol in Wireless Sensor Networks," Sensors, vol. 9, no. 1, pp. 445-462, 2009.

[24] D.-Y. Kim, J. Cho, and B. Lee, “A Buffer Management Technique for Guaranteed Desired Communication Reliability and LowPower in Wireless Sensor Networks," IEICE Transactions on Communications, vol. E93-B, no. 12, pp. 3522-3525, 2010. 